\title{
AMODAL COMPLETION OF BOUNDARIES IN COLOURED SURFACES
}

\author{
James DADAM, Liliana ALBERTAZZI'1), Luisa CANAL ${ }^{1)}$ and Rocco MICCIOLO ${ }^{1)}$ \\ ${ }^{1)}$ University of Trento, Italy
}

\begin{abstract}
We present an experimental study on the influence of colour and spatial position in the assessment of boundaries in occluded surfaces. Subjects were asked to mark the place that they judged to be the location of a boundary between two differently coloured parts of a vertical rectangle partially occluded by a horizontal rectangle. Besides the use of eight pairs of colours, the position of the occluder was randomly changed resulting in 13 possible sizes of the coloured parts. The same colours were set for half of the time on the lower part of the vertical rectangle, and for half on the upper part, so as to verify whether changing the colour location would give rise to measurable effects. Results show that amodal completion is influenced not only by spatial cues but also by colour.
\end{abstract}

Key words: amodal surfaces, amodal boundary, colour, completion

Completion is a widespread phenomenon in vision: it is the ability to perceive an object, in its entirety even though a part or some parts of it are perceived in the absence of the corresponding stimuli (Michotte, Thinès, \& Crabbé, 1964/1991, p. 144). In some cases the parts of the object are hidden by another entity, as in the case of occlusion, but observers are able to perceive the whole object, completing it amodally (Kanizsa, 1979, 1980, 1991; Koffka, 1935; Michotte et al., 1964/1991). In the context of natural selection, the phenomenon contributes to the simplicity and unity of visual objects, and therefore to the observer's biological survival (Ramachandran, 1987). Indeed, the perception of amodality is also present in non-humans, which confirms that it is a phenomenon which occurs at the primary process level (Aust \& Huber, 2006; Bakin, Nakayama, \& Gilbert, 2000; Deruelle, Barbet, Depy, \& Fagot, 2000; Fagot, Barbet, Parron, \& Deruelle, 2006; Kanizsa, Renzi, Conte, Compostela, \& Guerani, 1993; Kovàcs, Vogels, \& Orban, 1995; Miller, Dibble, \& Hauser, 2001; Nagasaka, Lazareva, \& Wasserman, 2007; Regolin \& Vallortigara, 1995; Regolin, Marconato, \& Vallortigara, 2004; Sovrano \& Bisazza, 2008; Tvardíková \& Fuchs, 2010). Given the pervasiveness of amodality in perception, its various aspects have been of constant interest to vision scientists, and there exists an extensive literature analysing the phenomenon from the point of view of bottom-up and top-down processes, its occurring with colour appearances, and with surface and face perception (see for example Gerbino \& Dalmaso, 1987, on amodal completion affecting pattern recognition; Anderson, Singh, \& Fleming, 2002; Fantoni, Gerbino, \& Kellman, 2008; and Yazdanbakhsh \& Watanabe, 2004, on amodal surfaces; Rauschenberger,

The authors thank the University Vale do Itajai for its collaboration during the conduct of this research, Prof. Osvaldo Da Pos for his assistance, and Massimo Vescovi for programming the data collection system.

Correspondence concerning this article should be addressed to Professor Liliana Albertazzi, CIMeC, University of Trento, Corso Bettini, 31, 38068 Rovereto (TN), ITALY (email: liliana.albertazzi@unitn.it). 
Peterson, Mosca, \& Bruno, 2004, on mosaic and completion stages; Lee \& Vecera, 2005, on the influence of higher level processes in visual completion; Albert, 2007; Anderson, 2007; and Kellman, Garrigan, Shipley, Yin, \& Machado, 2005; Kellman, Garrigan, Shipley, \& Keane, 2007, on interpolation processes; Pinna, 2008, on amodal completion of colour; Chen, Liu, Chen, \& Fang, 2009 on amodal completion in face perception; Takashima, Fujii, \& Shiina, 2009, on the influence of orientation and shape in completion).

Visual completion occurs not only in occlusion phenomena but also in those of contour interposition, apparent transparency (Glynn, 1954; Metelli, 1974; Rosenbach, 1902; Ware, 1980) or spontaneous figure splitting (Kanizsa, 1979; Koffka, 1935; Parks, 1986). The feature shared by these phenomena is the perception of depth or stratification. In this regard, Guibal and Dresp (2004) have underlined the role of colour, i.e., the fact that it can influence the perception of depth, although this in turn is strongly influenced by, for example, stimulus geometry, partial occlusion, contour interposition and positional cue, and luminance contrast. Furthermore, analyses of amodal completion can be grouped according to the factors responsible for their appearance, the methodologies used, and their assumptions, although these distinctions are not always clear-cut.

As to the first point, Tse (1999a) has categorised the theories that explain the way in which our visual system bridges the gaps generated by partially occluded objects into three groups. According to Tse (1999a) the first group comprises theories that use local image cues and maintain that completion occurs because of good continuation, contour orientation, and T-junction (Kellman \& Shipley, 1991; Takeichi, Nakazawa, Murakami, \& Shimojo, 1995; Wertheimer, 1923; Wouterlood \& Boselie, 1992). The second group considers the importance of global factors: symmetry, regularity, and simplicity of form or pattern are the cues used by our visual system to amodally complete occluded objects (Attneave \& Arnoult, 1956; Buffart, Leeuwenberg, \& Restle, 1981; Hochberg \& Brooks, 1960; Moravec \& Beck, 1986; Sekuler, 1994; Sekuler, Palmer, \& Flynn, 1994; van Lier, van der Helm, \& Leeuwenberg, 1994, 1995). Finally, the last group consists of theories about internal representations, these being traditionally surfaces (Nakayama \& Shimojo, 1992; Nakayama, Shimojo, \& He, 1995; Nakayama, Shimojo, \& Silverman, 1989), or more recently volumes (Tse, 1998, 1999b; Tse \& Albert, 1998; van Lier, 1999; van Lier \& Wagemans, 1999). In this last group, a recent approach proposed by Tse emphasizes the role of volume completion, in particular regard to the concept of mergeability, i.e. by intermediate representations such as volumes, a product of global image relationships (Tse, 1999a, p. 170).

As to the second point, the methodologies adopted may be mainly descriptive in line with classical experimental phenomenology (Michotte et al., 1964/1991; Kanizsa, 1991; Metelli, 1974), and/or psychophysical or neurophysiologic analysis (de Wit, Bauer, Oosterveld, Fries, \& van Lier, 2006; Hedgé, Fang, Murray, \& Kersten, 2008; Murray, Foxe, Javitt, \& Foxe, 2004; Plomp, Liu, van Leeuwen, \& Joannides, 2006; Rauschenberger, Liu, Slotnick, \& Yantis, 2006; Yazdanbakhsh \& Livingstone, 2006).

Psychophysical and neurophysiological analyses have often relied on computational models of vision, (for example, Marr \& Poggio, 1976; Pollard, Mayhew, \& Frisby, 1985; 
see Yin, Kellman, \& Shipley, 2000). Many researchers on perception, too, including some who pioneered information-theoretic approaches in cognitive science, have recently stressed the need for new conceptualizations and computational frameworks with which to analyze perceptual and mental processes (e.g. Hoffman, 2008a, 2008b; Richards, 1996; Richards, Rubin, \& Hoffman, 1983). Yet there have been no systematic attempts to bring together researchers in various disciplines who share these concerns, with the consequence that the problem remains generally unaddressed.

The choice of one method rather than another also depends on the problem that one wishes to address, so that the research focuses on different aspects of the same phenomenon and which contribute to its explanation, like: qualitative analysis of the percept and its conditions of appearance for the phenomenological-experimental method; analysis of the correlation between stimulus and behavioural response for psychophysics; and analysis of the correlates of neural activity for neuroscientific investigation. The different research options may usefully converge (Valberg, 2001; Valberg \& Lee, 1991). Kanizsa's analysis, for example, has provided excellent bases on which to determine neurophysiological correlates of amodal contours (Baumgartner, von der Heydt, \& Peterhans, 1984; De Weerd, Desimone, \& Underleider, 1998) while the Gestalt principle of Figure/Ground segregation has been important in the neuronal analysis of transparency phenomena (Qiu \& von der Heydt, 2005, 2007). Similarly, work on the perceptual significance of medial axis representations (Bouix, Siddiqui, Tannenbaum, \& Zucker, 2006; Leyton, 1987, 1992; van Tonder, Lyons, \& Ejima, 2002) suggests yet another avenue for neurophysiologic investigation. The problem arises mostly from the assumptions that guide the different options and/or from absolutist approaches to the data by the methodology adopted to explain and interpret the phenomenon. In fact, a certain barrier to communication in this regard still prevails in the community of vision scientists, although, as Spillmann (2009) has emphasised, descriptive, psychophysical, and neurophysiological investigations were all present in the Gestalt proposal, which assumed the existence of isomorphism among the structures at the various levels of organization.

\section{Colour Studies}

This paper is part of a research program examining the relationship between the appearance of colour and shape (Albertazzi et al., 2012; Dadam, Albertazzi, Da Pos, Canal, \& Micciolo, 2012). The paper itself is specifically devoted to an investigation of the role of colour with reference to occlusion. Colour analysis encounters a number of problems already mentioned in regard to the analysis of amodality - and to an even greater extent, given that colour is a characteristic of visual objects (be they surfaces or lights) of an eminently subjective nature. Classical psychophysical methodologies do not reveal the subjective appearance of colours, although they contribute to our understanding of some relationships among them.

In the vast majority of cases, underlying neural mechanisms are understood and studied as an explanation of perceived colour. In regard to the neurophysiological method, however, it should be pointed out that the use of neural correlates as explanatory criteria is still problematic (Malkoc, Kay, \& Webster, 2005). Moreover, perception by 
itself has a very loose relationship with the underlying physics of colour due to metamerism and other relational factors (Adelson, 1995).

The aim of this study is to test whether certain characteristics of colour can influence the balance or equilibrium in the division of a bi-coloured rectangle into its respective parts when the border between them is occluded by another rectangle and therefore seen amodally. In comparison with the mentioned literature, this study presents two aspects of novelty. Firstly, some perceptive attributes of colour, for instance their effect on the expansion/shrinkage and on the advancement/receding of surfaces, are phenomena of which painters and designers have always been aware of but that have received relatively little attention from scientists (Bailey, Grimm, \& Davoli, 2006; Chen \& Lin, 2005; Da Pos, 1988; De Valois \& De Valois, 1975; Egusa, 1983; Hering, 1878/1964; Jameson \& Hurvich, 1955; Katz, 1935; Luckiesh, 1918; Philipona \& O'Regan, 2006; Sivik, 1974; Tornquist, 1999). Secondly, as is well known, surfaces are decisive elements in determining the figural unity of visual objects (Yin, 1998; Yin et al., 2000); nevertheless, they are the least studied in the literature on amodality, which usually focuses on the role played by edge features in visual integration (Grossberg \& Mingolla, 1985; Kellman \& Shipley, 1991; Livingstone \& Hubel, 1987; Ramachandran, 1987; Yarbus, 1967). Correlative analyses on the neurocorrelates of amodal completion processes were not the focus of this research, although conducting them would be highly desirable.

One particular aspect of colour that is well known but caused a great deal of difficulty in the first theoretical studies is that of chromatic variations due to colour interaction (Albers, 2010). This phenomenon has been the subject of scientific studies: see, for example short and long ranging interactions (Spillmann \& Werner, 1996). The chromatic change is particularly obvious in the case of interactions between colours that are close to each other, and the first studies focussed on this-for instance, see Chevreul (1854) on simultaneous contrasts. Our research considers colour aspects and their relation to the amodal completion of partially occluded features. This problem may be described as a case of Percept-Percept coupling (Epstein, 1997), or in terms of how one perceptual size influences another-e.g. how perceived distance may influence perceived size (Gruber, 1954), or how perceived illumination may determine the perceived surface colour (Bergström, 2004). In this research it was checked whether particular phenomenal aspects of colour interaction that may be described in terms of contrast do in fact influence the figural organisation determined by amodal completion. In this case, the phenomenal contrast between colours did not occur by modal adjacency but from a distance, underneath the occluding rectangle and thus amodally. Even though the two coloured areas were perceptually seen as adjacent, they were separate at the physiological level and hence the amodality ought to have facilitated attribution of chromatic interaction to the phenomenal level.

\section{Dimensional and Colour Effects}

Dimensional effects such as shrinkage or expansion of the whole configuration are commonly observed in the presence of amodal completion (Kanizsa, 1972, 1975, 1980; Kanizsa \& Luccio, 1978; Luccio \& Edile, 1981; Tampieri, 1979; Vicario \& Tomat, 1991), 
although it is not certain that these effects result from amodal completion (Vezzani, 1994; Zanforlin, 1981). The hypothesis that we wanted to test was that the two areas of the rectangle bearing a different colour may appear of a different size depending on the colour combinations. Since one's expansion appears to occur at the same time as the other's shrinking, we wanted to see what kind of chromatic contrast may produce the two combined effects, i.e., what contrast produces the enlargement of an area and the contraction of another, and what position of the boundary is seen as the one that gives rise to a balanced division of the vertical rectangle (where by balanced is meant leading to two visually equal parts). It was tested whether the position of the margin chosen by the subjects as optimal division of the occluded rectangle was in agreement with the expectations based on the characteristics of the colours employed, i.e., whether a geometrically smaller area is chosen if its colour leads to its phenomenal expansion, and vice versa. It was also aimed to test whether the position of the occluder interferes with this determination.

The main chromatic contrast that was considered was that of lightness, so that the lighter area was expected to expand proportionally to its chromatic difference with the dark one. It was expected the difference to be greater with a black/white pair than with a pair of light/dark shades of grey. Secondly, another kind of contrast taken into consideration was that between warm and cool colours (Da Pos \& Valenti, 2007). We expected to find that if one colour was warmer than the other, it would correspond to a larger perceived area than its neighbour; for instance, it was expected red to determine a greater expansion than blue, at even lightness. It was also surmised that if the two colours contrast in apparent lightness, temperature and weight (light/heavy), the expansionary effect ought to be particularly marked. This is the case of the yellow/purple pair. It was therefore expected that the border would be moved to geometrically shrink the area that was seen as larger; if the two colours had equal effects of enlargement and shrinkage, it was assumed the margin would be seen as lying precisely in the centre. Another assumption was that the optimal perceptual equilibrium that can be realised in a rectangle divided into two parts is given by two parts of equal size.

In summary, the goal was to determine the colour of two differently coloured parts of a vertical rectangle acted on the perceived dimensions and thus, when subjects were asked to determine a balanced spatial relationship, to measure how they would bisect the rectangle based on various factors. It was hypothesized that this expectation also depended on the upper/lower position of each colour, since it seemed likely that by inverting the position of a pair of colours the results would not be symmetric. Indeed, if the dimensional relationship between the two areas depends essentially on the colours, then it should not depend on the position of the occluder. On the other hand, the occluder's position changes the relative dimensions of the exposed parts of the triangle, modally seen, and therefore one expects to see a tendency towards increasing the occluded area because the hidden part is perceived as being of smaller size.

The position of the occluder may interfere with the task of bisecting the vertical rectangle, since the occluder itself is subject to this bisection and interacts with the position of the bisecting margin as it is divided into two parts by the margin itself. One 
tends to either maintain the bar bisected as it is moved vertically, in which case the two areas of the vertical rectangle are subject to changes in their relative dimensions, or to maintain an equal division of the vertical rectangle so that the occluder appears divided into parts of unequal size, whose relationship varies as a function of its position. Clearly, the viewer's attention and subjective disposition can play a significant role in this, depending on their focus while they carry out the task.

\section{METHOD}

\section{Participants}

The experiment was conducted in Brazil with a total of 30 subjects, mainly with undergraduate and postgraduate students from the University of the Vale do Itajai (Santa Catarina). The overall mean age was 28.8. There were no major demographic or social differences among subjects which might have given rise to differences in the results.

\section{Stimuli and Apparatus}

The experiment was carried out in a room with constant and controlled lighting conditions (the luminance on the table, lit at 400 lux, was about $45 \mathrm{~cd} / \mathrm{m}^{2}$ ). Luminance and colorimetric measurements of the colours are given in Table 1. The subjects were seated at a desk in front of a DELL Latitude D630. The observer was positioned so that they would be looking at the centre of the screen perpendicularly from a distance of $60 \mathrm{~cm}$. A chin rest was not used to keep the subject at the correct distance, but we ensured that the subjects adopted the correct posture on the chair, guaranteeing the perpendicularity of the angle of vision with respect to the screen, as well as the pre-established distance. The brightness and contrast of the screen were unchanged for all experiments, and the computer was mains powered to avoid any shifts in brightness due to power shortages. An opaque screen was used and the ambient light source was not shining on the screen. The stimuli were presented on the aforementioned screen: they consisted of a vertical $10 \times 3.5 \mathrm{~cm}(9.5 \times 3.34$ $\mathrm{deg}$ ) rectangle, divided into two parts of different colours - one upper, the other lower-and of a pale orangecoloured horizontal $2 \times 6 \mathrm{~cm}(1.9 \times 5.7 \mathrm{deg})$ rectangle that occluded the boundary region between the two colours of the vertical rectangle.

We decided to use a simple stimulus with relatable contours and surfaces very similar to Fig. 1a in Tse (1999a, p. 166). The horizontal occluding rectangle was displayed in 13 different positions, always occluding the boundary region between the two colours. The screen centre and the vertical rectangle centre coincided at the location termed ' 0 ', which set the reference point for every other vertical position mentioned in what follows, with positive numbers above and negative numbers below. Since the position of the occluder could only change discretely (pixel by pixel) and on the vertical axis, its position will always be referred to by using pixels as a measurement unit (corresponding to about $0.023 \mathrm{deg}$ ). In the central position, the centre of the occluder coincided with the centre of the vertical rectangle and the centre of the screen. The occluding rectangle was drawn in six positions above, and six below, the centre, for a total of 13 positions at regular intervals of $0.16 \mathrm{deg}$. In the highest position, the occluding rectangle's upper border was fixed at $1.91 \mathrm{deg}$ and the lower border at $-0.023 \mathrm{deg}$. The second highest horizontal rectangle had its upper border at $1.75 \mathrm{deg}$ and the lower border at $-0.18 \mathrm{deg}$. Other horizontal rectangle coordinates were: $1.59 /-0.34 \mathrm{deg}, 1.43 /-0.50$ deg, 1.27/-0.66 deg, 1.11/-0.82 deg, 0.95/-0.98 deg, 0.79/-1.13 deg, 0.64/-1.29 deg, 0.48/-1.45 deg, 0.32/$1.61 \mathrm{deg}, 0.16 /-1.77 \mathrm{deg}, 0.00 /-1.93 \mathrm{deg}$. The background was beige coloured.

Eight colour combinations were used, always combined in upper/lower pairs: the achromatic pair white and black, and the reverse, as the pair that manifests the highest lightness contrast; light grey and dark grey, and the reverse, where the lightness contrast is still achromatic but lower; the chromatic pair red and blue, and the reverse, manifesting the contrast of perceived temperature; yellow and purple, and the reverse, manifesting a contrast of lightness, temperature, apparent weight and apparent spatial dimension (see Table 1 for colour values). Hues were chosen in their nuance more significant, i.e. the high chromaticness, and the other chromatic attributes (lightness, whiteness, blackness and colourfulness) are consequential to this choice. The organization of the colours has also followed the concept of harmonic and disharmonic configurations of 
Table 1. Colours and Relative Measurement

\begin{tabular}{lrrr}
\hline \multicolumn{1}{c}{ Colour } & \multicolumn{1}{c}{$\mathrm{L}^{*}$} & $\mathrm{a}^{*}$ & $\mathrm{~b}^{*}$ \\
\hline White $\left(155 \mathrm{~cd} / \mathrm{m}^{2}\right)$ & 100.00 & -9.21 & 17.07 \\
Black & 9.52 & -0.01 & 1.61 \\
Light grey & 77.47 & -8.65 & 1.04 \\
Dark grey & 43.58 & -4.54 & -3.23 \\
Yellow & 92.83 & -9.47 & 76.78 \\
Purple & 30.68 & 19.69 & -21.05 \\
Red & 55.11 & 58.06 & 57.51 \\
Blue & 47.89 & -1.26 & -56.10 \\
Beige (background) & 88.40 & -4.98 & 25.78 \\
Pale orange (occluder) & 76.07 & 15.16 & 37.86 \\
\hline
\end{tabular}

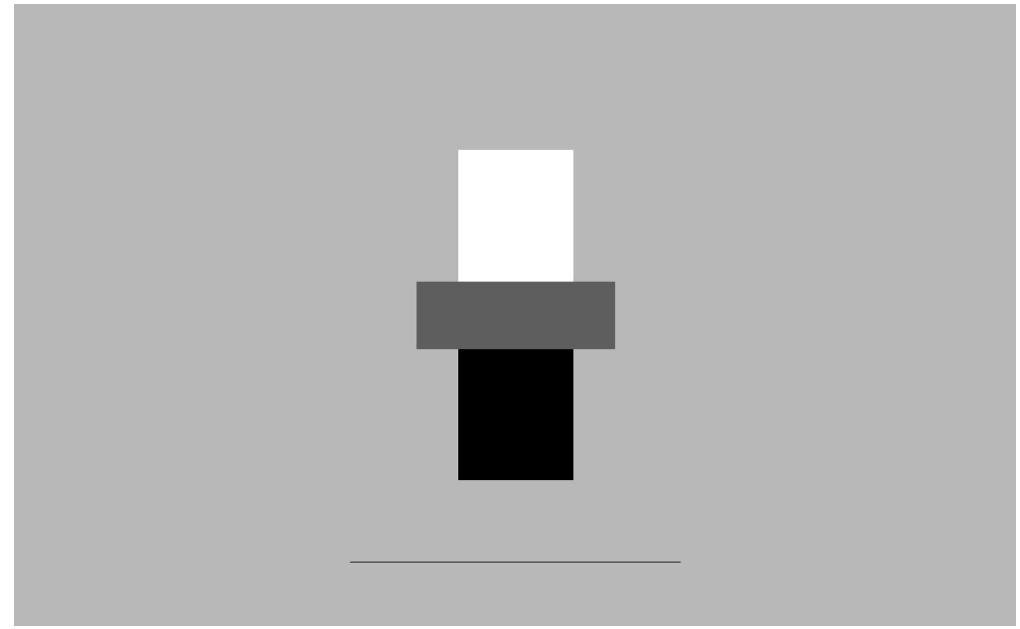

Fig. 1. Stimulus with occluder in the central position. Occluder and background colours have been changed here.

colour (Bullough, 1907; Da Pos, 1999), in which a harmonic configuration is composed of light colours (white, light grey, red and yellow) on the upper part of the configuration, and the dark colours (black, dark grey, blue and purple) on the bottom part. According to Bullough, dark colours appear heavier and should stay on the bottom part in order to maintain the balance of the configuration.

The combination of eight pairs of colours and 13 occluding positions of the horizontal rectangle yielded a total of 104 stimuli, which were shown randomly to each subject, who gave just one answer for each stimulus. Each confirmed answer corresponded to an exact position on the stimulus. Furthermore, a black line was displayed sometimes above, sometimes below the vertical rectangle. Fig. 1 shows the stimulus with the horizontal rectangle in central position $(0.95 /-0.98 \mathrm{deg})$. 
Procedure

It was assumed that the unitary nature of the rectangle that follows from the force of collinearity of the vertical margins remains even when the two uncovered areas are of a different colour. Consequently, there will be only one margin, amodally seen behind the horizontal rectangle, separating the two colours. An analogous phenomenon is the paradox of Kanizsa's figures, in which the unitary nature of the animal given by the continuation of the occluded margins makes the viewer see a very different animal from the one that may be described from our experience (Kanizsa, 1991, Chapter 2).

The subjects' task was to identify the perceptual boundary between the two coloured parts of the vertical rectangle. They were told that two coloured parts of the vertical rectangle were contiguous behind the horizontal rectangle, and that there would be a unique boundary somewhere. Subjects were also told that the colour of the parts, the position of the occluding rectangle, and the dimensions of both parts of the vertical rectangle could change in the different presentations. Finally, they were asked to mark the boundary's location by shifting the black line provided, which could be moved up and down using the mouse or keyboard. Subjects could change the position until they were satisfied; they then pressed 'enter/return' to record their answer. After each answer, a new stimulus was presented until the end of the experiment. There was no time limit. No information was given about the presumed expanding/shrinking effects on the surfaces due to the colours. Each subject saw different random presentations of the 104 stimuli.

Instructions given to the subjects:

Each test begins with the presentation of a rectangle with two different colours, partially covered by a second smaller rectangle. Above or below the largest rectangle you will also see a black line. The boundary between the two colours lies in the occluded zone.

Your task is to decide where the boundary lies perceptively between the two coloured parts of the rectangle. Use the mouse or the UP and DOWN arrow keys to move the black line and position it where you think that the boundary lies between the two colours. When you are sure of your answer press the ENTER key to confirm it. You will then be immediately presented with a new stimulus to evaluate.

Take all the time necessary for your choice. The position of the line can be changed as long as the ENTER key is not pressed. Try to be as accurate as possible.

The task given to the subjects was therefore purely phenomenological, based on subjective judgments. The instructions were given by the same researcher for all the subjects. After each subject had been tested, they were asked to describe their rationale.

\section{Experimental Design}

The research adopted a factorial design; two factors were investigated (position with 13 levels, and pair of colours with 8 levels). Each combination of position and pair of colours was randomly proposed to each subject. There were consequently 104 repeated measures within each subject. The results were analysed by means of a two-way analysis of variance with repeated measures on both factors-position and pair of colours, both evaluated as fixed effects - and considering subjects as a random effect. A significance level of 0.05 was always adopted. Post hoc comparisons among means were performed employing the Bonferroni correction.

\section{RESULTS}

The aim of the experiment was to determine whether the position of the occluded margin as a divisor of the vertical rectangle into two parts depended on the characteristics of the colours and was conditioned by the position of the occluding rectangle. Both these effects were highly statistically significant; the $\mathrm{F}$ test for the position of the occluding rectangle was $9.15(d f=12,348 ; p<0.001)$ while the $\mathrm{F}$ test for the pair of colours was $3.89(d f=7,203 ; p<0.001)$; on the other hand the interaction was not significant $(F=1.21 ; d f=84,2436 ; p=0.093)$. The mean values of the perceptual boundary position, shown in Fig. 2, were between -0.292 and +0.299 (overall mean +0.039 ), and the 


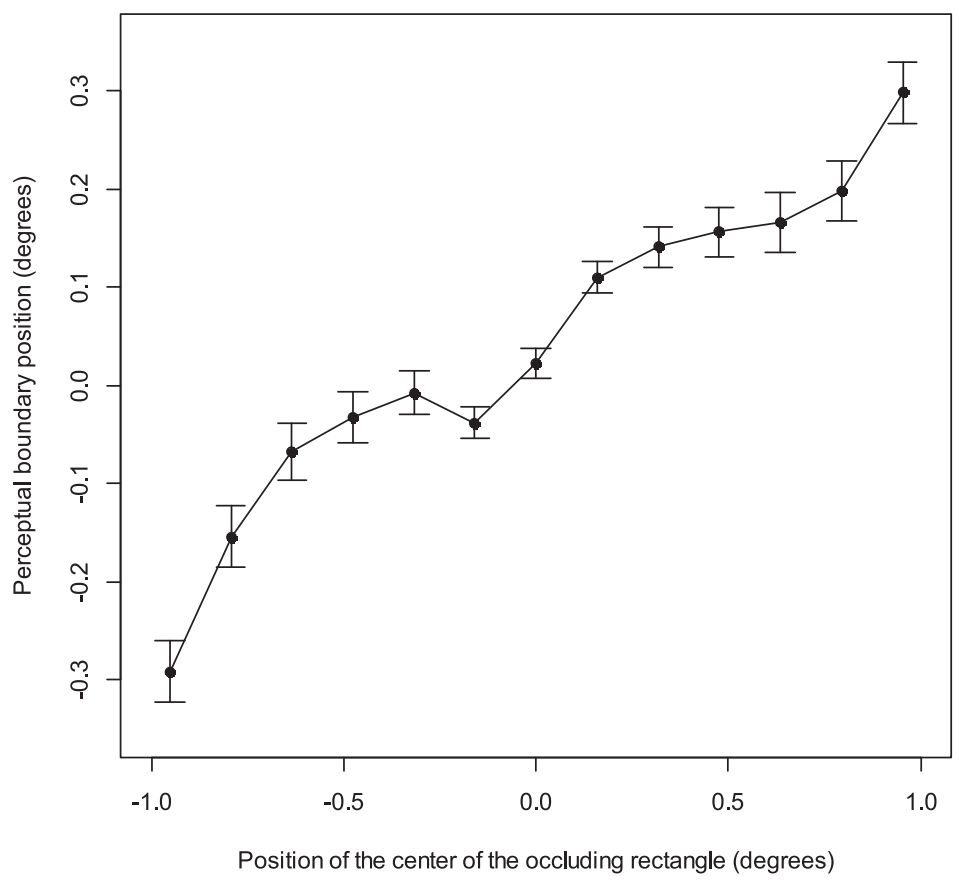

Fig. 2. Mean values (and standard errors) of the position of the perceptual boundaries in relation to the 13 uniformly scaled positions of the occluding rectangle. Values on the vertical axis have the centre of the screen (i.e., the centre of the vertical rectangle) as the origin.

standard deviation of all the 3120 measures was 0.426 .

Fig. 2 shows the mean values (together with their standard errors) of the position of the perceptual boundaries assigned by the subjects, taking the centre of the screen (i.e., the centre of the vertical rectangle) as the origin. Located on the horizontal axis are the 13 positions of the occluding rectangle, ranging from the lowest on the left to the highest on the right. It will be seen that the pattern present in Fig. 2 does not have a linear trend. Subjects tended to place the perceptual boundary in the region close to the centre of the screen (and of the vertical rectangle), with somewhat greater shifts only when the occluding rectangle was either very low (position furthest to the left in the figure) or very high (position furthest to the right in the figure).

Two phenomena are noticeable in the subjects: the first is that they tended to halve the vertical rectangle, and the second is that when the occluding rectangle got too close to the upper or lower positions, subjects tended to place the bar boundary in the lower half and the upper half of the occluding rectangle, respectively. When asked about the reasons for their choice, the subjects replied that, given the presentation of the image, a symmetrical configuration, where the boundary remained at the centre of the vertical rectangle, seemed more natural to them. Thus, regardless of the position of the occluder, the two coloured parts of the vertical rectangle had to be of the same size. Many of the subjects said that they had looked for the centre of the vertical rectangle and were sure that 


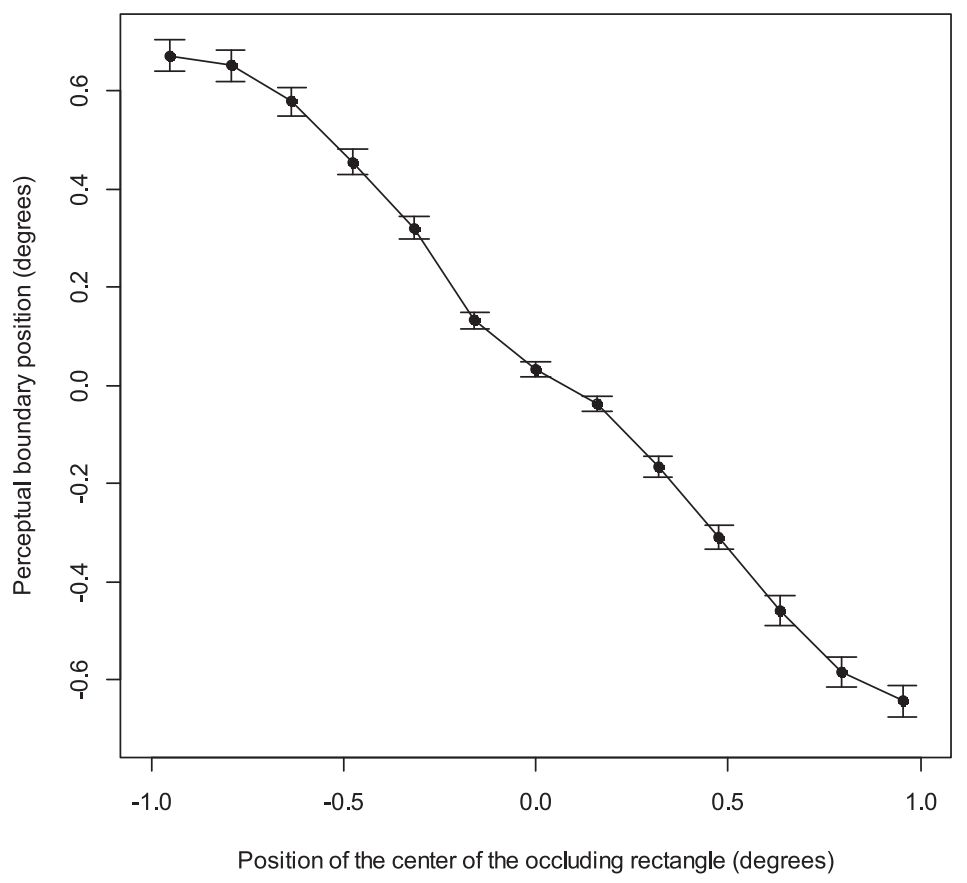

Fig. 3. Mean values (and standard errors) of the position of the perceptual boundaries in relation to the 13 uniformly scaled positions of the occluding rectangle. Values on the vertical axis have the centre of the occluding rectangle as the origin.

they had found it in the great majority of cases.

The perturbations introduced by the position of the occluder explain the discrepancy between the perception of equilibrium in the subjects and the geometric division that might have been expected in theory. The data shows that this (in the sense of an exact measure) happened very infrequently, despite the 'certainty' of the participants that they had identified the centre, giving the same dimension to both coloured parts. It is very likely that, despite the subjects' belief that they had found a position of perfect symmetry, they had failed to do so. In other words, the boundary was identified according to the 'centre' of the perceptual dimensions, not the physical ones.

If we consider as the origin the centre of the occluding rectangle instead of the centre of the vertical rectangle, we obtain Fig. 3. Here, the zero on the vertical axis corresponds to the centre of the occluder, while located on the horizontal axis are the 13 positions of the occluding rectangle.

The means have a much larger range than that displayed in Fig. 2. These results do not bear out the hypothesis that the task of the subjects was to search for the boundary with respect to the occluding rectangle rather than the occluded one. If the occluding rectangle moves downwards (the six positions on the left), the border is assigned to the upper half of the occluder; that is, closer to the centre than to the lower border. But if the occluding rectangle moves upwards (the six positions to the right), the boundary is 
Table 2. Mean Values and Standard Deviations (Degrees) of the Perceptual Boundary Position According to the Combination of Colours. Values Reported Have the Centre of the Screen (i.e., of the Vertical Rectangle) as the Origin.

\begin{tabular}{llll}
\hline \multicolumn{2}{c}{ Pair of colours } & & Mean \\
\cline { 1 - 2 } Upper & Lower & & SD \\
White & Black & 0.060 & 0.45 \\
Black & White & -0.015 & 0.40 \\
Light grey & Dark grey & 0.087 & 0.44 \\
Dark grey & Light grey & 0.024 & 0.42 \\
Yellow & Purple & 0.070 & 0.42 \\
Purple & Yellow & 0.033 & 0.43 \\
Red & Blue & 0.038 & 0.41 \\
Blue & Red & 0.014 & 0.44 \\
\hline
\end{tabular}

assigned to the lower half of the occluding rectangle, always closer to the centre than to the upper border.

One might expect to find that, the lower the position of the occluder, the lower the perceptual boundary assigned, so that the upper part, modally larger, becomes even more extensive, while the lower, modally smaller one, does not advance significantly into the amodal space. This expectation would arise from studies based on the contour support ratio or the proportion of image-specified contours (Fantoni \& Gerbino, 2003). In fact, "The ratio principle (Shipley \& Kellman, 1992) states that the good continuation absolute strength depends on the ratio between lengths of the image-specified portion and the total side (including the amodal portion predicted by good continuation alone). Good continuation becomes stronger as the specified length increases relative to the total length (i.e., when the support ratio is large)" (Fantoni \& Gerbino, 2003, p. 299).

According to this principle, the longer the contour, the greater becomes the extrapolation of the contour in the amodal space, so that, in our case, the boundary should move towards the shortest contour. However, research data show exactly the opposite: the part which has the smaller modal area tends to occupy a larger area in the occluded space than does the larger part; this in turn protrudes less into the amodal area. As mentioned above, a significant effect of the pair of colours was also found. Table 2 shows the mean values of the perceptual boundary according to the eight pairs of colours.

Four post-hoc comparisons were performed within the sample between the white/ black, black/white pairs, between the light grey/dark grey, dark grey/light grey pairs, between the yellow/purple, purple/yellow pairs, and between the red/blue, blue/red pairs, using the Bonferroni correction. Two significant differences were found when mean values of white/black and black/white were compared $(p=0.007)$ and when mean values of light grey/dark grey and dark grey/light grey were compared $(p=0.030)$. On the other 
hand, none of the yellow/purple, purple/yellow and red/blue, blue/red pairs showed significant differences $(p>0.4)$.

Furthermore, two other post-hoc comparisons were performed. The first, which compared achromatic and chromatic groups of colours, was not significant $(p>0.5)$; in fact, the two means were quite similar: $+0.038 \mathrm{deg}$ for the achromatic groups of colours and $+0.039 \mathrm{deg}$ for the chromatic groups of colours. The second comparison was made between harmonic and disharmonic groups of colours. In this case a significant result $(p<0.001)$ was found: the mean for harmonic colours was $+0.064 \mathrm{deg}$, while for disharmonic ones it was $+0.014 \mathrm{deg}$. In other words, it can be explained on the basis of the difference in the modally present quantity of each area and its colour. The type of colour, in fact (for example yellow), may have exerted an effect of perceptive expansion on one of the areas, with the consequent shrinking of the other, provoking a perceptual impression of the dimensions different from the 'real' ones. Consequently, the boundary was identified according to the 'centre' of the perceptual dimensions, not the physical ones. As regards the colours, it was shown that the choice of position for the separating line depends on the chromatic conditions whenever the results obtained for a couple of colours are the opposite of those obtained by reversing their relative positions (top/ bottom). The difference in the results shows that the positioning was due to chromatic characteristics.

\section{DISCUSSION}

The research analysed the relationship between a situation of amodality of surfaces in the case of occlusion and the role of colour in comparison to spatial cues. The subjects' task was to identify the best place for the perceptual boundary between the two coloured parts of a vertical rectangle occluded by a smaller horizontal rectangle. As previously mentioned, we expected to find some effects in the amodal perception of boundaries due to some characteristics of colour (light/heavy, expanding/shrinking, harmonic/ disharmonic), and some due to the relative position (lower/upper) of the coloured parts of the occluded rectangle. The results show that boundary perception of amodality in occluded surfaces can be influenced by colour characteristics. Furthermore, the results show that subjects tended to place the boundary in relation to the position (upwards or downwards) occupied by the occluding rectangle and there is a tendency to place the boundary closer to the centre of the vertical rectangle.

When asked about their choice after the test, in most cases the subjects replied that they were trying to find the symmetry between the two coloured parts of the vertical rectangle. Most of them, independently of the occluder's position, perceived the two differently coloured parts of the vertical rectangle as having the same dimension. Their choice can be explained in terms of the second group of theories identified by Tse (1999a), in which completion occurs when certain conditions among global image cues are fulfilled: for example, symmetry, regularity or simplicity. If so, the choice made by the subjects to prefer a symmetric configuration rather than using other cues, such as the 
extension of both modal parts of the vertical rectangle, to decide where boundaries could be placed would also confirm Michotte's (1964/1991) hypothesis, i.e., that a global property of the whole is involved in the process and not a completion restricted to the amodal area. Of course, it is not possible to exclude that their choice may also have involved a top-down operation: according to the stimulus that they saw, the subjects may first have decided that the boundaries had to be placed in the centre and subsequently calculated where the centre should be. If they did proceed in this way, the results show that although some subjects reportedly attempted to place the boundary in the centre of the vertical rectangle, in most cases they were unable to locate the metric centre, although some of them were convinced that they had done so. The effects of different extensions and hues of the coloured parts probably induced some of the subjects to perceive the central position in a place different from the metric centre of the vertical rectangle.

For 7 out of the 8 pairs of colours considered, the colour located in the lower part seemed to expand, putting the boundary in the upper part of the vertical rectangle, as if the lower part had to be bigger in order to sustain the upper part. The means reported in Table 2 show that the dark colours — black, dark grey, purple and blue- had a higher expansion than the light colours. This is in agreement with Claessen, Overbeeke, \& Smets (1995): light colours seemingly occupy a larger area than the dark colours, although they have the same extension of area. Since subjects seem to prefer a more regular and symmetrical configuration, in order to compensate the apparent larger area of light colour, they tend to expand more dark colours areas (i.e., the heavier) in order to find a balance between both colours.

Several studies have been carried out in the field of colour aesthetics, and it is widely believed that harmony and balance are related (Da Pos, 1999). Bullough (1907) uses the concept of balance in the gravitational sense: dark colours are heavier than light colours because they have an apparent 'moreness'; thus, to achieve a balance, the heavier colour should stand below the lighter one. Other studies have confirmed the assumption that dark colours are seen as heavier than light colours (DeCamp, 1917; Monroe, 1926; Payne, 1958, 1961; Taylor, 1930; Warden \& Flynn, 1926). In this way, a harmonic configuration would be composed of a dark and heavier colour on the lower part, and a light and lighter colour on the upper part.

Interesting results were found when groups of harmonic and disharmonic colours were compared. The subjects preferred to expand the lower part of the vertical rectangle, with a larger expansion of the dark coloured parts (for instance purple: when it was on the lower part, the boundary was placed at +0.070 deg above the centre, expanding the purple area and shrinking the yellow one, while in the opposite configuration, with yellow in the lower part, the boundary was placed at $+0.033 \mathrm{deg}$ above the centre, expanding the yellow area, but not as in purple case). It seems that both the spatial configuration below/above (the lower part had to be bigger since seven of the eight lower parts expanded with the exception of white, which shrank) and the colour configuration mattered in order to find a balance (dark colours had to expand more than light colours in order to compensate for the apparently larger area of the light colours). We found a significant difference between the white/black and black/white configurations and between the light grey/dark grey and dark 
grey/light grey configurations. It is likely that the black part expanded because it was seen as much smaller than the white one, with a bigger expansion when it was on the lower part of the vertical rectangle; on the other hand, both greys expanded when they were on the lower part of the vertical rectangle, with a bigger expansion for the dark grey. It would be interesting to see whether these results also hold with a horizontal configuration.

In the case of the apparent weight of some colours, there are some discrepancies among studies: for example, the results are not sufficiently consistent to assume that blue is heavier than red, since in some experiments the opposite has been found (Payne, 1958; Warden \& Flynn, 1926). However, as Wright (1962) noted, in most cases studies on the apparent warmth and weight of colours do not consider all the three basic perceptual dimensions of colour - hue, brightness and saturation — so that this may be the cause of the discrepancies among researches in this field. This author points that DeCamp (1917), and Payne (1958), Taylor (1930) and Warden and Flynn (1926), do not take saturation into consideration and the effects of hue and lightness were confounded in their analysis.

In this study, the background was always of a beige colour. It would be interesting to evaluate the role of different backgrounds; there are studies which have found an interaction between the effect of colour and the effect of background brightness (Chen $\&$ Lin, 2005; Egusa, 1983; Guibal \& Dresp, 2004). As already mentioned, Guibal and Dresp (2004), for instance, have observed that the background may change the perception of depth. In one of their experiments, studying colour contrast of isoluminant red, green and white, they found that red figures were perceived as 'nearer' when presented on a light background, while white and green figures were seen as 'nearer' with a dark background.

In conclusion, this paper has shown that amodal completion is influenced not only by spatial cues but also by colour. Although in most cases the subjects have preferred a symmetrical solution, this arrangement varied in function of the position of the colours (upper/lower) and their characteristics, such as light/dark. The colours on the lower part of the vertical rectangle tended to expand, with a higher expansion for dark colours building harmonic configurations.

\section{REFERENCES}

Adelson, E. H. 1995. Checkershadow Illusion. Available in http://web.mit.edu/persci/people/adelson/ checkershadow_illusion.html

Albers, J. 2010. Interaction of Colours. Revised and Expanded Edition. London: Yale University Press.

Albert, M. K. 2007. Mechanisms of modal and amodal interpolation. Psychological Review, 114, 455-469.

Albertazzi, L., Da Pos, O., Malfatti, M., Micciolo, R., Canal, L., \& Vescovi, M. 2012. The hue of shapes. Journal of Experimental Psychology: Human Perception and Performance. DOI: 10.1037/a0028816

Anderson, B. L. 2007. The demise of the identity hypothesis and the insufficiency and nonnecessity of contour relatability in predicting object interpolation: Comment on Kellman, Garrigan, and Shipley (2005). Psychological Review, 114, 470-487.

Anderson, B. L., Singh, M., \& Fleming, R. W. 2002. The interpolation of object and surface structure. Cognitive Psychology, 44, 148-190.

Attneave, F., \& Arnoult, M. D. 1956. The quantitative study of shape and pattern perception. Psychological Bulletin, 53, 452-471.

Aust, U., \& Huber, L. 2006. Does the use of natural stimuli facilitate amodal completion in pigeons? 
Perception, 35, 333-349.

Bailey, R. J., Grimm, C. M., \& Davoli, C. 2006. The real effect of warm-cool colors. Tech. Rep. 17, Washington University in St. Louis. Available in http://wwwl.cse.wustl.edu/ cmg/content/papers/ tech200617/tech200617.pdf

Bakin, J. S., Nakayama, K., \& Gilbert, C. D. 2000. Visual responses in monkey areas V1 and V2 threedimensional surface configurations. Journal of Neuroscience, 20, 8188-8198.

Baumgartner, G., von der Heydt, R., \& Peterhans, E. 1984. Anomalous contours: A tool of studying the neurophysiology of vision. Experimental Brain Research, Suppl 9, 413-419.

Bergström, S. S. 2004. The AMBEGUJAS phenomenon and colour constancy. Perception, 33, 831-835.

Bouix, S., Siddiqui, K., Tannenbaum, A., \& Zucker S. 2006. Medial axis computation and evolution. In H. Krim \& A. Yezzi (Eds.), Statistics and Analysis of Shapes, Modeling and Simulation in Science. Basel-Boston-Berlin: Birkhauser Verlag.

Buffart, H., Leeuwenberg, E., \& Restle, F. 1981. Coding theory of visual pattern completion. Journal of Experimental Psychology: Human Perception and Performance, 7, 241-274.

Bullough, E. 1907. On the apparent heaviness of colours. A contribution to the aesthetic of colour. British Journal of Psychology, 2, 111-152.

Chen, I., \& Lin, J. 2005. Quantitative assessment of the advancing/receding and the expansion/constriction effects of colours. Proceedings of the $10^{\text {th }}$ Congress of the International Colour Association, Granada, Spain, 1481-1484.

Chen, J., Liu, B., Chen, B., \& Fang, F. 2009. Time course of amodal completion in face perception. Vision Research, 49, 732-758.

Chevreul, M. E. 1854. The Principles of Harmony of Colours and Contrast of Colours, and Their Applications to the Arts. London: Longmans.

Claessen, J. P., Overbeeke, C. J., \& Smets, G. J. F. 1995. Puzzling colours. Color Research and Application, 20, 388-396.

Da Pos, O. 1988. Natural lightness of hues and chromostereopsis. Colour Dynamics, 88, Budapest, Hungary.

Da Pos, O. 1999. Sull'armonia del colore [About the colour harmony]. In L. Lorenzi (Ed.), Il pensiero della bellezza. Milan, Italy: Franco Angeli.

Da Pos, O., \& Valenti, V. 2007. Warm and cold colours. In Y. Guanrong \& X. Haisong (Eds.), AIC Colour Science for Industry. Hangzhou, China: Color Association of China.

Dadam, J., Albertazzi, L., Da Pos, O., Canal, L., \& Micciolo, R. 2012. Morphological patterns and their colour. Perceptual \& Motor Skills, 114, 363-377.

De Valois, R. L., \& De Valois, K. K. 1975. Neural coding of colour. In E. C. Carterette \& M. P. Friedman (Eds.), Handbook of Perception. New York: Academic Press.

De Weerd, P., Desimone, R., \& Underleider, L. G. 1998. Perceptual filling-in: a parametric study. Vision Research, 38, 2721-2734.

de Wit, T. C. J., Bauer, M., Oosterveld, R., Fries, P., \& van Lier, R. 2006. Cortical responses to contextual influences in amodal completion. NeuroImage, 32, 1815-1825.

DeCamp, J. E. 1917. The influence of color in apparent weight: A preliminary study. Journal of Experimental Psychology, 2, 347-370.

Deruelle, C., Barbet, I., Depy, D., \& Fagot, J. 2000. Perception of partly occluded figures by baboons (Papio papio). Perception, 39, 1483-1497.

Egusa, H. 1983. Effects of brightness, hue and saturation on perceived depth between adjacent regions in the visual field. Perception, 12, 167-175.

Epstein, W. 1997. Percept-Percept couplings. In E. Rock (Ed.), Indirect Perception. Cambridge, MA: MIT Press.

Fagot, J., Barbet, I., Parron, C., \& Deruelle, C. 2006. Amodal completion by baboons (Papio papio): contribution of background depth cues. Primates, 47, 145-150.

Fantoni, C., \& Gerbino, W. 2003. Contour interpolation by vector-field combination. Journal of Vision, 3, 281-303.

Fantoni, C., Gerbino, W., \& Kellman, P. J. 2008. Approximation, torsion, and amodally-completed surfaces. Vision Research, 48, 1196-1216.

Gerbino, W., \& Dalmaso, F. 1987. The effect of amodal completion on visual matching. Acta Psychologica, 65, 25-46. 
Glynn, I. M. 1954. Linked sodium and potassium movements in human red cells. The Journal of Physiology, 126, 35 .

Grossberg, S., \& Mingolla, E. 1985. Neural dynamics of form perception: boundary completion, illusory figures, and neon color spreading. Psychological Review, 92, 173-211.

Gruber, H. E. 1954. The relation of perceived size to perceived distance. The American Journal of Psychology, 67, 411-426.

Guibal, C. R. C., \& Dresp, B. 2004. Interaction of color and geometric cues in depth perception: When does "red" mean "near"? Psychological Research, 69, 30-40.

Hedgé, J., Fang, F., Murray, S. O., \& Kersten, D. 2008. Preferential responses to occluded objects in the human visual cortex. Journal of Vision, 8, 1-16.

Hering, E. 1964. Outlines of a Theory of the Light Sense (L. M. Hurvich \& D. Jameson, Trans.). Cambridge, MA: Harvard University Press. (Original work published 1878)

Hochberg, J., \& Brooks, V. 1960. The psychophysics of form: Reversible-perspective drawings of spatial objects. American Journal of Psychology, 73, 337-354.

Hoffman, D. D. 2008a. Sensory experiences as cryptic symbols of a multi-modal user interface. In M. Bauer, F. Liptay \& S. Marschall (Eds.), Kunst und Kognition. Munich, Germany: Wilhelm Fink.

Hoffman, D. D. 2008b. Conscious realism and the mind-body problem. Mind and Matter, 6, 87-121.

Jameson, D., \& Hurvich, L. M. 1955. Some quantitative aspects of an opponent-colors theory. I. Chromatic responses and spectral saturation. Journal of the Optical Society of America, 45, 546-552.

Kanizsa, G. 1972. Schrumpfung von visuellen Feldern bei amodaler Ergänzung. Studia Psychologica, 14, 208-210.

Kanizsa, G. 1975. Amodal completion and phenomenal shrinkage of surfaces in the visual field. Italian Journal of Psychology, 2, 187-195.

Kanizsa, G. 1979. Organization in Vision: Essays on Gestalt Perception. New York: Praeger.

Kanizsa, G. 1980. Grammatica del vedere [Grammar of Seeing]. Bologna, Italy: Il Mulino.

Kanizsa, G. 1991. Vedere e pensare [Seeing and Thinking]. Bologna, Italy: Il Mulino.

Kanizsa, G., \& Luccio, R. 1978. Espansione di superficie da completamento amodale [Expansion of amodal completion surfaces]. Reports from the Institute of Psychology. University of Trieste, Italy.

Kanizsa, G., Renzi, P., Conte, S., Compostela, C., \& Guerani, L. 1993. Amodal completion in mouse vision. Perception, 22, 713-721.

Katz, D. 1935. The World of Colour. London: Routledge.

Kellman, P. J., \& Shipley, T. F. 1991. A theory of visual interpolation in object perception. Cognitive Psychology, 23, 141-221.

Kellman, P. J., Garrigan, P., Shipley, T. F., \& Keane, B. P. 2007. Interpolation processes in object perception: reply to Anderson (2007). Psychological Review, 114, 488-508.

Kellman, P. J., Garrigan, P., Shipley, T. F., Yin, C., \& Machado, L. 2005. 3-d interpolation in object perception: Evidence from an objective performance paradigm. Journal of Experimental Psychology: Human Perception and Performance, 31, 558-583.

Koffka, K. 1935. Principles of Gestalt Psychology. New York: Harcourt Brace.

Kovàcs, G., Vogels, R., \& Orban, G. A. 1995. Selectivity of macaque inferior temporal neurons for partially occluded shapes. Journal of Neuroscience, 15, 1984-1997.

Lee, H., \& Vecera, S. P. 2005. Visual cognition influences early vision: The role of visual short-term memory in amodal completion. Psychological Science, 16, 763-768.

Leyton, M. 1987. Symmetry-Curvature duality. Computer Vision, Graphics, and Image Processing, 38, 327-341.

Leyton, M. 1992. Symmetry, Causality, Mind. Cambridge, MA: MIT Press.

Livingstone, M. S., \& Hubel, D. H. 1987. Psychophysical evidence for separate channels for the perception of form, color, movement, and depth. Journal of Neuroscience, 7, 3416-3468.

Luccio, R. \& Edile, A. L. 1981. Il completamento amodale di superfici amodali [The amodal completion of amodal surfaces]. Giornale Italiano di Psicologia, 8, 141-148.

Luckiesh, M. 1918. On retiring and advancing colors. American Journal of Psychology, 29, 182-186.

Malkoc, G., Kay, P., \& Webster, M. A. 2005. Variations in normal color vision. IV. Binary hues and hue scaling. Journal of the Optical Society of America, 22, 2154-2168.

Marr, D., \& Poggio, T. 1976. Cooperative computation of stereo disparity. Science, 194, 283-287. 
Metelli, F. 1974. Achromatic color conditions in the perception of transparency. In R. B. MacLeod \& H. L. Pick Jr. (Eds.), Perception: Essays in Honour of James J. Gibson. Ithaca, NY: Cornell University Press.

Michotte, A., Thinès, G., \& Crabbé, G. 1991. Les compléments amodaux des structures perceptives. In G. Thinès, A. Costall, \& G. Butterworth (Eds.), Michotte's Experimental Phenomenology of Perception. Hillsdale, NJ: Lawrence Erlbaum. (Original work published 1964)

Miller, C. T., Dibble, E., \& Hauser, M. D. 2001. Amodal completion of acoustic signals in a nonhuman primate. Nature Neuroscience, 4, 783-784.

Monroe, M. 1926. The apparent weight of color and correlated phenomena. The American Journal of Psychology, 36, 192-206.

Moravec, L., \& Beck, J. 1986. Amodal completion: Simplicity is not the explanation. Bulletin of the Psychonomic Society, 24, 269-272.

Murray, M. M., Foxe, D. M., Javitt, D. C., \& Foxe, J. J. 2004. Setting boundaries: Brain dynamics of modal and amodal illusory shape completion in humans. The Journal of Neuroscience, 24, 6898-6903.

Nagasaka, Y., Lazareva, O. F., \& Wasserman, E. A. 2007. Prior experience affects amodal completion in pigeons. Perception \& Psychophysics, 69, 596-605.

Nakayama, K., \& Shimojo, S. 1992. Experiencing and perceiving visual surfaces. Science, 257, 1357-1363.

Nakayama, K., Shimojo, S., \& He, Z. J. 1995. Visual surface representation: A critical link between lowerlevel and higher-level vision. In S. M. Kosslyn \& D. N. Osherson (Eds.), Visual Cognition: An Invitation to Cognitive Science (Vol. 2, 2nd ed.). Cambridge, MA: MIT Press.

Nakayama, K., Shimojo, S., \& Silverman, G. H. 1989. Stereoscopic depth: Its relation to image fragmentation, grouping, and the recognition of occluded objects. Perception, 18, 55-68.

Parks, T. E. 1986. Illusory figures, illusory objects, and real objects. Psychological Review, 93, 207-215.

Payne, M. C. 1958. Apparent weight as a function of color. The American Journal of Psychology, 71, 725730 .

Payne, M. C. 1961. Apparent weight as a function of hue. The American Journal of Psychology, 74, 104105.

Philipona, D. L., \& O'Regan, J. K. 2006. Colour naming, unique hues, and hue cancellation predicted from singularities in reflection properties. Visual Neuroscience, 23, 331-339.

Pinna, B. 2008. A new perceptual problem: the amodal completion of color. Visual Neuroscience, 25, 415422.

Plomp, G., Liu, L., van Leeuwen, C., \& Joannides, A. A. 2006. The 'Mosaic Stage' in amodal completion as characterized by magnetoencephalography responses. Journal of Cognitive Science, 18, 1394-1405.

Pollard, S. B., Mayhew, J. E., \& Frisby, J. P. 1985. PMF: a stereo correspondence algorithm using a disparity gradient limit. Perception, 14, 449-470.

Qiu, F. T., \& von der Heydt, R. 2005. Figure and ground in the visual cortex: V2 combines stereoscopic cues with Gestalt rules. Neuron, 47, 155-166.

Qiu, F. T., \& von der Heydt, R. 2007. Neural representation of transparent overlay. Nature Neuroscience, 10, 283-284.

Ramachandran, V. S. 1987. Visual perception of surfaces: a biological theory. In P. S. Meyer (Ed.), The Perception of Illusory Contours. Berlin, Germany: Springer.

Rauschenberger, R., Liu, T., Slotnick, S. D., \& Yantis, S. 2006. Temporally unfolding neural representation of pictorial occlusion. Psychological Science, 17, 358-364.

Rauschenberger, R., Peterson, M. A., Mosca, F., \& Bruno, N. 2004. Amodal completion in visual search: Preemption or context effects? Psychological Science, 15, 351-355.

Regolin, L., Marconato, F., \& Vallortigara, G. 2004. Hemispheric differences in the recognition of partly occluded objects by newly hatched domestic chicks (Gallus gallus). Animal Cognition, 7, 162-170.

Regolin, L., \& Vallortigara, G. 1995. Perception of partly occluded objects by young chicks. Perception \& Psychophysics, 57, 971-976.

Richards, W. A. 1996. Priors by design. In D. Knill \& W. Richards (Eds.), Perception as Bayesian Inference. Cambridge, MA: Cambridge University Press.

Richards, W. A., Rubin, J. M., \& Hoffman, D. D. 1983. Equation counting and the interpretation of sensory data. Perception, 11, 557-576.

Rosenbach, O. 1902. Zur Lehre von den Urtheilstäuschungen. Zeitschrift für Psychologie, 29, 434-448. 
Sekuler, A. B. 1994. Local and global minima in visual completion: Effects of symmetry and orientation. Perception, 23, 529-545.

Sekuler, A. B., Palmer, S. E., \& Flynn, C. 1994. Local and global processes in visual completion. Psychological Science, 5, 260-267.

Shipley, T. F., \& Kellman, P. J. 1992. Strength of visual interpolation depends on the ratio of physically specified to total edge length. Perception \& Psychophysics, 52, 97-106.

Sivik, L. 1974. Color meaning and perceptual color dimensions. A study of color samples. Göteborg Psychological Reports, 4, No. 1.

Sovrano, V. A., \& Bisazza, A. 2008. Recognition of partly occluded objects by fish. Animal Cognition, 11, $161-166$.

Spillmann, L. 2009. Phenomenology and neurophysiological correlations: Two approaches to perception research. Vision Research, 49, 1507-1521.

Spillmann, L., \& Werner, J. S. 1996. Long range interactions in visual perception. Trends in Neurosciences, 19, 428-434.

Takashima, M., Fujii, T., \& Shiina, K. 2009. Amodal completion is not completed only behind the occluder. Perception, 38, 1410-1412.

Takeichi, H., Nakazawa, H., Murakami, I., \& Shimojo, S. 1995. The theory of the curvature-constraint line for amodal completion. Perception, 24, 373-389.

Tampieri, G. 1979. The shrinkage of amodal completed surfaces in figure-ground situation. Italian Journal of Psychology, 6, 53-57.

Taylor, C. D. 1930. Visual perception versus visual plus kinaesthetic perception in judging colored weights. Journal of General Psychology, 4, 229-246.

Tornquist, J. 1999. Colore e luce: Teoria e pratica [Colour and Light: Theory and Practice]. Milan, Italy: Istituto del Colore.

Tse, P. U. 1998. Illusory volumes from conformation. Perception, 27, 977-993.

Tse, P. U. 1999a. Complete mergeability and amodal completion. Acta Psychologica, 102, 165-201.

Tse, P. U. 1999b. Volume completion. Cognitive Psychology, 39, 37-68.

Tse, P. U., \& Albert, M. K. 1998. Amodal completion in the absence of image tangent discontinuities. Perception, 27, 455-464.

Tvardíková, K., \& Fuchs, R. 2010. Tits use amodal completion in predator recognition: A field experiment. Animal Cognition, 13, 609-615.

Valberg, A. 2001. Unique hues: An old problem for a new generation. Vision Research, 41, 1645-1657.

Valberg, A., \& Lee, B. B. 1991. From pigments to perception: Advances in understanding visual processes. NATO ASI Series, Series A, life sciences, Vol. 203. New York: Plenum.

van Lier, R. J., \& Wagemans, J. 1999. From images to objects: Global and local completions of self-occluded parts. Journal of Experimental Psychology: Human Perception and Performance, 25, 1721-1741.

van Lier, R. J., van der Helm, P. A., \& Leeuwenberg, E. L. J. 1994. Integrating global and local aspects of visual occlusion. Perception, 23, 883-903.

van Lier, R. J., van der Helm, P. A., \& Leeuwenberg, E. L. J. 1995. Competing global and local completions in visual occlusion. Journal of Experimental Psychology: Human Perception and Performance, 21, 571-583.

van Lier, R. J. 1999. Investigating global effects in visual occlusion: From a partly occluded square to the back of a tree-trunk. Acta Psychologica, 102, 203-220.

van Tonder, G. J., Lyons, M. J., \& Ejima, Y. 2002. Visual structure of a Japanese Zen garden. Nature, 419, 359-360.

Vezzani, S. 1994. La contrazione fenomenica: un tentativo di confutazione sperimentale della spiegazione di Kanizsa fondata sul completamento amodale [The phenomenal contraction: an attempt to experimental confutation of Kanizsa explanation based on amodal completion]. Ricerche di Psicologia, 18, 95-109.

Vicario, G. B., \& Tomat, L. 1991. Un caso di contrazione apparente di spazi [A case of apparent contraction of spaces]. Contributi di Psicologia, 4, 125-140.

Warden, C. J., \& Flynn, E. L. 1926. The effect of color on apparent size and weight. The American Journal of Psychology, 37, 398-401.

Ware, C. 1980. Coloured illusory triangles due to assimilation. Perception, 9, 103-107.

Wertheimer, M. 1923. Untersuchungen zur Lehre von der Gestalt. Psychologische Forschung, 4, 301-350. 
Wouterlood, D., \& Boselie, F. 1992. A good-continuation model of some occlusion phenomena. Psychological Research/Psychologische Forschung, 54, 267-277.

Wright, B. 1962. The influence of hue, lightness, and saturation on apparent warmth and weight. The American Journal of Psychology, 75, 232-241.

Yarbus, A. L. 1967. Eye Movements and Vision. (B. Haigh, Trans.). New York: Plenum.

Yazdanbakhsh, A., \& Livingstone, M. S. 2006. End stopping in V1 is sensitive to contrast. Nature Neuroscience, 9, 697-702.

Yazdanbakhsh, A., \& Watanabe, T. 2004. Asymmetry between horizontal and vertical illusory lines in determining the depth of their embedded surfaces. Vision Research, 44, 2621-2627.

Yin, C. 1998. A task-oriented taxonomy of visual completion. Behavioral and Brain Sciences, 21, 780-781.

Yin, C., Kellman, P. J., \& Shipley, T. F. 2000. Surface integration influences depth discrimination. Vision Research, 40, 1969-1978.

Zanforlin, M. 1981. Perception of apparent width using adjacent surfaces. Part I. Amodal completion and surface shrinkage (Report 31). Istituto di Psicologia, University of Padova, Italy.

(Manuscript received 4 March, 2011; Revision accepted 2 July, 2012) 